Int. J. Contemp. Math. Sciences, Vol. 2, 2007, no. 27, 1335 - 1340

\title{
A Note on Browkin-Brzezinski's Conjecture
}

\author{
Nguyen Thanh Quang \\ Department of Mathematics, Vinh University \\ Vinh, Nghe An, Vietnam \\ Phan Duc Tuan \\ Department of Mathematics, Vinh University \\ Vinh, Nghe An, Vietnam \\ tuanphanduc@yahoo.com
}

\begin{abstract}
In this paper we prove Browkin-Brzezinski's conjecture for a class of polynomials.
\end{abstract}

Keywords: abc-Theorem, Browkin-Brzezinski's conjecture, Wronskian

\section{Introduction}

Let $F$ be a fixed algebraically closed field of characteristic 0 . Let $f(z)$ be a polynomial non - constants with coefficients in $F$ and let $r(f)$ be the number of distinct zeros of $f$. Then we have the following.

Mason's theorem ([3]). Let $a(z), b(z), c(z)$ be relatively prime polynomials in $F$ and not all constants such that $a+b=c$. Then

$$
\max \{\operatorname{deg}(a), \operatorname{deg}(b), \operatorname{deg}(c)\} \leq r(a b c)-1 .
$$

For different purposes some generalizations of the theorem are given (see $[2,4])$. In [1], Browkin and Brzezinski suggested the following conjecture.

Conjecture 1.1 Let $f_{0}, \ldots, f_{n+1}$ be $n+2$ polynomials not all constants in $F[x]$ and have no common zeros such that

$$
f_{0}+\cdots+f_{n}+f_{n+1}=0 .
$$

Then

$$
\max _{0 \leq j \leq n+1} \operatorname{deg}\left(f_{j}\right) \leq(2 n-1)\left(r\left(f_{0} \cdots f_{n+1}\right)-1\right) .
$$

In this paper we prove the following theorem.

Theorem 1.1 Browkin-Brzezinski's conjecture is true for polynomials $f_{0}, \ldots, f_{n+1}$ such that $g d c\left(f_{i}, f_{j}, f_{k}\right)=1$ for all distinct $i, j, k \in\{0, \ldots, n+1\}$. 


\section{Proof of the main theorem}

Let $f$ is a rational function, we write $f$ in the form:

$$
f=\frac{f_{1}}{f_{2}}
$$

Where $f_{1}, f_{2}$ are polynomial functions are non-zero and relatively prime on $F[x]$. The degree of $f$, denoted by $\operatorname{deg} f$, is define to be $\operatorname{deg} f_{1}-\operatorname{deg} f_{2}$.

Let $a \in F$, we write $f$ in the form:

$$
f=(x-a)^{l} \frac{g_{1}}{g_{2}}
$$

and $g_{1}(a) g_{2}(a) \neq 0$, then $l$ is called the order of $f$ at $a$ and is denoted by $\mu_{f}^{a}$. We have the following easily proved properties of $\mu_{f}^{a}$.

Lemma 2.1 Let $f, g$ be two polynomials and $a \in F$, we have

a) $\mu_{f+g}^{a} \geq \min \left(\mu_{f}^{a}, \mu_{g}^{a}\right)$,

b) $\mu_{f g}^{a}=\mu_{f}^{a}+\mu_{g}^{a}$,

c) $\mu_{\frac{f}{g}}^{a}=\mu_{f}^{a}-\mu_{g}^{a}$,

Lemma 2.2 Let $\varphi$ be a the rational function on $F$ and let the derivatives order $k$ of $\varphi$ satisfy the following $\varphi^{(k)} \not \equiv 0$. Then

$$
\mu_{\varphi(k)}^{\alpha} \geq-k+\mu_{\varphi}^{\alpha}
$$

Proof. Let $\varphi(x)=(x-\alpha)^{m} \frac{f(x)}{g(x)}$, where $f(x), g(x)$ are relatively prime and $f(\alpha) g(\alpha) \neq 0$. Then, we have

$$
\varphi^{\prime}(x)=(x-\alpha)^{m-1} \frac{m f(x) g(x)+(x-\alpha)\left(f^{\prime}(x) g(x)-f(x) g^{\prime}(x)\right)}{g^{2}(x)} .
$$

By $\mu_{g}^{\alpha}=0$, we have

$$
\mu_{\varphi^{\prime}}^{\alpha} \geq m-1
$$

Therefore

$$
\mu_{\varphi^{\prime}}^{\alpha} \geq-1+\mu_{\varphi}^{\alpha}
$$

From this we obtain

$$
\mu_{\varphi(k)}^{\alpha} \geq-k+\mu_{\varphi}^{\alpha}
$$

Lemma 2.3 Browkin-Brzezinski's conjecture is true for polynomials $f_{0}, \ldots, f_{n+1}$ such that $g d c\left(f_{i}, f_{j}, f_{k}\right)=1$ for all distinct $i, j, k \in\{0, \ldots, n+1\}$ and $f_{0}, \ldots, f_{n}$ are linearly independent. 
Proof. By the hypothesis $f_{0}, \ldots, f_{n}$ are linearly independent, we have the Wronskian $W$ of $f_{0}, \ldots, f_{n}$ does not vanish. We set

$$
\begin{aligned}
P & =\frac{W\left(f_{0}, \ldots, f_{n}\right)}{f_{0} \ldots f_{n}}, \\
Q & =\frac{f_{0} \ldots f_{n+1}}{W\left(f_{0}, \ldots, f_{n}\right)} .
\end{aligned}
$$

Hence we have

$$
f_{n+1}=P Q
$$

We first prove that

$$
\operatorname{deg} Q \leq(2 n-1) r\left(f_{0} \cdots f_{n+1}\right)
$$

Suppose that $\alpha$ is a zero of $f_{0} f_{1} \cdots f_{n+1}$, by the hypothesis there exists $\nu, 0 \leq \nu \leq n+1$ such that $f_{\nu} \neq 0$. By the hypothesis $f_{0}+\cdots+f_{n}=f_{n+1}$ we have

$$
\begin{aligned}
\mu_{\frac{f_{0} \cdots f_{n+1}}{W\left(f_{0}, \ldots, f_{n}\right)}}^{\alpha} & =\mu_{\frac{f_{0} \cdots f_{\nu-1} f_{\nu+1} \cdots f_{n+1}}{W\left(f_{0}, \ldots, f_{\nu-1}, f_{\nu+1}, \ldots, f_{n+1}\right)}}^{\alpha} \\
& =\sum_{j=0}^{n+1} \mu_{f_{j}}^{\alpha}-\mu_{W\left(f_{0}, \ldots, f_{\nu-1}, f_{\nu+1}, \ldots, f_{n+1}\right)}^{\alpha}
\end{aligned}
$$

$W\left(f_{0}, \ldots, f_{\nu-1}, f_{\nu+1}, \ldots, f_{n+1}\right)$ is the sum of follow terms

$$
\delta f_{\alpha_{0}}\left(f_{\alpha_{1}}\right)^{\prime} \cdots\left(f_{\alpha_{n}}\right)^{(n)}
$$

Where $\alpha_{i} \in\{0, \ldots n+1\} \backslash\{\nu\}, \delta= \pm 1$. We suppose there exists $k$ functions $f_{j}$ such that $f_{j}(\alpha)=0$, . By the hypothesis $g d c\left(f_{i}, f_{j}, f_{k}\right)=1$ for all distinct $i, j, k \in\{0, \ldots, n+1\}$ we have $k \leq 2$. From this and Lemma 2.1 and we have

$$
\begin{aligned}
& \mu_{\left.f_{\alpha_{0}}\left(f_{\alpha_{1}}\right)^{\prime \cdots\left(f_{\alpha_{n}}\right.}\right)^{(n)}}^{\alpha} \\
\geq & \sum_{f_{j}(\alpha)=0} \mu_{f_{\alpha_{j}}}^{\alpha}-(n+(n-1)) \\
= & \mu_{\prod_{j=0}^{n+1} f_{\alpha_{j}}}^{\alpha}-(2 n-1)
\end{aligned}
$$

By Lemma 2.2 we have

$$
\mu_{W\left(f_{0}, \ldots, f_{\nu-1}, f_{\nu+1}, \ldots f_{n+1}\right)}^{\alpha} \geq \mu_{\prod_{j=0}^{n+1} f_{\alpha_{j}}}^{\alpha}-(2 n-1) .
$$

Hence

$$
\mu_{\frac{f_{0} \cdots f_{n+1}}{W\left(f_{0}, \ldots, f_{n}\right)}}^{\alpha} \leq 2 n-1
$$


By the definition of degree of a rational function, we have:

$$
\operatorname{deg} Q \leq(2 n-1) r\left(f_{0} \cdots f_{n+1}\right) .
$$

Next, we will prove that

$$
\operatorname{deg} P \leq-\frac{n(n+1)}{2} .
$$

At here, we have $P$ as the logarithmic Wronskian corresponding to $I=$ $\{0,1, . ., n\}$ which is

$$
\left|\begin{array}{cccc}
1 & 1 & \ldots & 1 \\
\frac{f_{0}^{\prime}}{f_{0}} & \frac{f_{1}^{\prime}}{f_{1}} & \ldots & \frac{f_{n}^{\prime}}{f_{n}} \\
\vdots & \vdots & \ddots & \vdots \\
\frac{f_{0}^{(n)}}{f_{0}} & \frac{f_{1}^{(n)}}{f_{1}} & \ldots & \frac{f_{n}^{(n)}}{f_{n}}
\end{array}\right|
$$

The determinant $P$ is a summa of following terms

$$
\delta \frac{f_{\beta_{1}}^{\prime} f_{\beta_{2}}^{\prime \prime} \ldots f_{\beta_{n}}^{(n)}}{f_{\beta_{1}} f_{\beta_{2}} \ldots f_{\beta_{n}}} .
$$

For every term, we have

$$
\begin{gathered}
\operatorname{deg}\left(\frac{f_{\beta_{1}}^{\prime} f_{\beta_{2}}^{\prime \prime} \ldots f_{\beta_{n}}^{(n)}}{f_{\beta_{1}} f_{\beta_{2}} \ldots f_{\beta_{n}}}\right)=\operatorname{deg}\left(\frac{f_{\beta_{1}}^{\prime}}{f_{\beta_{1}}}\right)+\operatorname{deg}\left(\frac{f_{\beta_{2}}^{\prime \prime}}{f_{\beta_{2}}}\right)+\ldots+\operatorname{deg}\left(\frac{f_{\beta_{n}}^{(n)}}{f_{\beta_{n}}}\right) \\
=-(1+2+\ldots+n)=-\frac{n(n+1)}{2} .
\end{gathered}
$$

Therefore

$$
\operatorname{deg} P \leq-\frac{n(n+1)}{2} .
$$

From (2), (3), (4) we have

$$
\begin{aligned}
\operatorname{deg} f_{n+1} & =\operatorname{deg} P+\operatorname{deg} Q \leq(2 n-1) r\left(f_{0} \cdots f_{n+1}\right)-\frac{n(n+1)}{2} \\
& \leq(2 n-1)\left(r\left(f_{0} \cdots f_{n+1}\right)-1\right) .
\end{aligned}
$$

Similar arguments apply to the polynomial $f_{0}, f_{1}, \ldots, f_{n}$, we have

$$
\max _{0 \leq 1 \leq n+1}\left(\operatorname{deg} f_{i}\right) \leq(2 n-1)\left(r\left(f_{0} \cdots f_{n+1}\right)-1\right) .
$$

Proof of theorem 1.1. The proof proceed by induction on $n$. For $n=1$, it is true by Mason's theorem. Suppose that the theorem is true for all case $m, 1 \leq m \leq n-1$. If $f_{0}, \ldots, f_{n}$ are linearly independent, then this is Lemma 2.3. If $f_{0}, \ldots, f_{n}$ are linearly dependent, rewriting (1) as

$$
-f_{n+1}=f_{0}+\cdots+f_{n},
$$


Let $f_{i_{1}}, \ldots, f_{i_{q}}, q<n+1$ be a maximal linearly independent subset of the $f_{j}, j=$ $0, \ldots, n$, since $n \geq 1$ and $g d c\left(f_{i}, f_{j}, f_{k}\right)=1$ for all distinct $i, j, k \in\{0, \ldots, n+1\}$ it follows that $q \geq 2$. Then each $f_{j}, 0 \leq j \leq n, j$ not one of $i_{k}$, is a linear combination of the $f_{i_{k}}$, of the form

$$
f_{j}=\lambda_{1} f_{j_{1}}+\cdots+\lambda_{q} f_{i_{q}}
$$

where the $\lambda_{k} \in F$, and at least two of these $\lambda_{k}$ are not zero. Using our inductive hypothesis we apply the theorem to (6). This yields that if $\lambda_{k} \neq 0$ then

$$
\operatorname{deg}\left(f_{i_{k}}\right) \leq(2 q-1)\left(r\left(f_{j} \prod_{k=1}^{q} f_{i_{k}}\right)-1\right)
$$

So that

$$
\operatorname{deg}\left(f_{i_{k}}\right) \leq(2 n-1)\left(r\left(\prod_{k=0}^{n+1} f_{k}\right)-1\right)
$$

From (6) the same estimate as in (8) follows for $\operatorname{deg} f_{j}$. Thus the theorem is proved for such $f_{j}$ and $f_{i_{k}}$. Inserting all the relations of the form (6) into the right side of (5) yields an equation of the form

$$
f_{n+1}=\kappa_{1} f_{j_{1}}+\cdots+\kappa_{q} f_{i_{q}}
$$

where the $\kappa_{j} \in F$. Moreover, if one of these $\kappa_{v}=0$ then the corresponding $f_{i_{v}}$ must have appeared in one of the equations (6) with a non-zero $\lambda_{v}$. Hence (7) has been established for this $f_{i_{v}}$. Finally, for those $\kappa_{v} \neq 0$, we treat (9) exactly as we did (6), (note that $q+1<n+1$ ), and obtain the estimate (8) for $\operatorname{deg} f_{i_{v}}$, and for $\operatorname{deg} f_{n+1}$. This completes the induction.

\section{References}

[1] Browkin, J. and Brzezinski, J., Some remarks on the abc conjecture, Mathematics of Computation, 62, (1994) 931-939.

[2] P.C. Hu and C.C.Yang, Notes on a generalized abc-conjecture over function fields, Ann. Math. Blaise Pascal 8 (2001), no. 1, 61-71.

[3] Lang, S., Old and new conjectured Diophantine inequalities, Bull. Amer. Math. Soc. 23 (1990), 37?75.

[4] Leonid N. Vaserstein and Ethel R. Wheland, Vanishing polynomial sums, Communications in Algebra, 31, No. 2, (2003), 751-772.

[5] Mason, R. C., Equations over function fields, Lecture Notes in Math. 1068 (1984), 149-157, Springer. 
[6] Mason, R.C., Diophantine equations over function fields, London Math. Soc. Lec-ture Note Ser. 96, Cambridge Univ. Press, Cambridge, 1984.

[7] Nguyen Thanh Quang and Phan Duc Tuan, Siu-Yeng's lemma in the padic case, Vietnam Journal of Mathematics, 32:2, No.2 (2004), 227-234.

[8] H.N. Shapiro and G.H.Sparer, Extension of a Theorem of Mason, Commm. Pure and Appl. Math., 47 (1994), 711-718.

Received: May 11, 2007 